\title{
Hermite-Hadamard type inequalities for $n$-times differentiable and preinvex functions
}

\author{
Shu-Hong Wang ${ }^{1 *}$ and Feng Qi ${ }^{1,2,3}$
}

"Correspondence:

shuhong7682@163.com

${ }^{1}$ College of Mathematics, Inner Mongolia University for

Nationalities, Tongliao City, Inner Mongolia Autonomous Region

028043, China

Full list of author information is

available at the end of the article

\begin{abstract}
In the paper, by creating an integral identity involving an $n$-times differentiable function, the authors establish some new Hermite-Hadamard type inequalities for preinvex functions and generalize some known results.
\end{abstract}

MSC: Primary 26D15; secondary 26A51; 26B12; 41A55; 49J52

Keywords: integral identity; Hermite-Hadamard type inequality; preinvex function

\section{Introduction}

Throughout this paper, let $\mathbb{R}=(-\infty, \infty)$ and $\mathbb{N}$ denote the set of all positive integers.

Let us recall some definitions of various convex functions.

Definition 1 A function $f: I \subseteq \mathbb{R} \rightarrow \mathbb{R}$ is said to be convex if

$$
f(\lambda x+(1-\lambda) y) \leq \lambda f(x)+(1-\lambda) f(y)
$$

holds for all $x, y \in I$ and $\lambda \in[0,1]$. If the inequality (1) reverses, then $f$ is said to be concave on $I$.

Definition 2 [1] A set $S \subseteq \mathbb{R}^{n}$ is said to be invex with respect to the map $\eta: S \times S \rightarrow \mathbb{R}^{n}$, if $y+t \eta(x, y) \in S$ for every $x, y \in S$ and $t \in[0,1]$.

It is obvious that every convex set is invex with respect to the map $\eta(x, y)=x-y$, but there exist invex sets which are not convex. See [1], for example.

Definition 3 [1] Let $S \subseteq \mathbb{R}^{n}$ be an invex set with respect to $\eta: S \times S \rightarrow \mathbb{R}^{n}$. For every $x, y \in S$, the $\eta$-path $P_{x v}$ joining the points $x$ and $v=x+\eta(y, x)$ is defined by

$$
P_{x v}=\{z \mid z=x+\operatorname{t\eta }(y, x), t \in[0,1]\} .
$$

Definition 4 [1] Let $S \subseteq \mathbb{R}^{n}$ be an invex set with respect to $\eta: S \times S \rightarrow \mathbb{R}^{n}$. A function $f: S \rightarrow \mathbb{R}$ is said to be preinvex with respect to $\eta$, if $f(y+t \eta(x, y)) \leq t f(x)+(1-t) f(y)$ for every $x, y \in S$ and $t \in[0,1]$.

Every convex function is preinvex with respect to the map $\eta(x, y)=x-y$, but not conversely. For properties and applications of preinvex functions, please refer to [1-3] and closely related references therein.

O2014 Wang and Qi; licensee Springer. This is an Open Access article distributed under the terms of the Creative Commons Attribution License (http://creativecommons.org/licenses/by/2.0), which permits unrestricted use, distribution, and reproduction in any medium, provided the original work is properly cited. 
The most important inequality in the theory of convex functions, the well-known Hermite-Hadamard's integral inequality, may be stated as follows. If $f$ is a convex function on $[a, b]$, then

$$
f\left(\frac{a+b}{2}\right) \leq \frac{1}{b-a} \int_{a}^{b} f(x) \mathrm{d} x \leq \frac{f(a)+f(b)}{2}
$$

If $f$ is concave on $[a, b]$, then the inequality (3) is reversed.

The inequality (3) has been generalized by many mathematicians. Some of them may be recited as follows.

Theorem 1 [4, Theorem 2.2] Let $f: I^{\circ} \subseteq \mathbb{R} \rightarrow \mathbb{R}$ be a differentiable mapping on $I^{\circ}$ and $a, b \in I^{\circ}$ with $a<b$. If $\left|f^{\prime}(x)\right|$ is convex on $[a, b]$, then

$$
\left|\frac{f(a)+f(b)}{2}-\frac{1}{b-a} \int_{a}^{b} f(x) \mathrm{d} x\right| \leq \frac{(b-a)\left[\left|f^{\prime}(a)\right|+\left|f^{\prime}(b)\right|\right]}{8} .
$$

Theorem 2 [5, Theorem 1] Iff is differentiable on $[a, b]$ such that $\left|f^{\prime}(x)\right|^{q}$ is a convex function on $[a, b]$ for $q \geq 1$, then

$$
\left|\frac{f(a)+f(b)}{2}-\frac{1}{b-a} \int_{a}^{b} f(x) \mathrm{d} x\right| \leq \frac{b-a}{4}\left[\frac{\left|f^{\prime}(a)\right|^{q}+\left|f^{\prime}(b)\right|^{q}}{2}\right]^{1 / q} .
$$

Theorem 3 [6, Theorem 2.3] Let $f: I \rightarrow \mathbb{R}$ be differentiable on $I^{\circ}, a, b \in I^{\circ}$ with $a<b$, and $p>1$. If $\left|f^{\prime}(x)\right|^{p /(p-1)}$ is convex on $[a, b]$, then

$$
\begin{aligned}
\left|f\left(\frac{a+b}{2}\right)-\frac{1}{b-a} \int_{a}^{b} f(x) \mathrm{d} x\right| \\
\quad \leq \frac{b-a}{16}\left(\frac{4}{p+1}\right)^{1 / p}\left\{\left[\left|f^{\prime}(a)\right|^{p /(p-1)}+3\left|f^{\prime}(b)\right|^{p /(p-1)}\right]^{1-1 / p}\right. \\
\left.\quad+\left[3\left|f^{\prime}(a)\right|^{p /(p-1)}+\left|f^{\prime}(b)\right|^{p /(p-1)}\right]^{1-1 / p}\right\} .
\end{aligned}
$$

Theorem 4 [2, Theorem 2.1] Let $A \subseteq \mathbb{R}$ be an open invex set with respect to $\eta: A \times A \rightarrow \mathbb{R}$ and $f: A \rightarrow \mathbb{R}$ be a differentiable function. If $\left|f^{\prime}(x)\right|$ is preinvex on $A$, then for every $a, b \in A$ with $\eta(a, b) \neq 0$

$$
\left|\frac{f(b)+f(b+\eta(a, b))}{2}-\frac{1}{\eta(a, b)} \int_{b}^{b+\eta(a, b)} f(x) \mathrm{d} x\right| \leq \frac{|\eta(a, b)|}{8}\left[\left|f^{\prime}(a)\right|+\left|f^{\prime}(b)\right|\right] .
$$

Theorem 5 [2, Theorem 4.1] Let $A \subseteq \mathbb{R}$ be an open invex set with respect to $\eta: A \times A \rightarrow \mathbb{R}$ and $\eta(a, b) \neq 0$ for all $a \neq b$. Suppose that $f: A \rightarrow \mathbb{R}$ is a twice differentiable function on $A$. If $f^{\prime \prime}(x) \mid$ is preinvex on $A$ and $f^{\prime \prime}$ is integrable on the $\eta$-path $P_{b c}$ for $c=b+\eta(a, b)$, then

$$
\left|\frac{f(b)+f(b+\eta(a, b))}{2}-\frac{1}{\eta(a, b)} \int_{b}^{b+\eta(a, b)} f(x) \mathrm{d} x\right| \leq \frac{[\eta(a, b)]^{2}}{24}\left[\left|f^{\prime \prime}(a)\right|+\left|f^{\prime \prime}(b)\right|\right] .
$$

Theorem 6 [2, Theorem 4.3] Let $A \subseteq \mathbb{R}$ be an open invex set with respect to $\eta: A \times A \rightarrow \mathbb{R}$ and $\eta(a, b) \neq 0$ for all $a \neq b$. Suppose that $f: A \rightarrow \mathbb{R}$ is a twice differentiable function on $A$ 
and $\left|f^{\prime \prime}(x)\right|$ is preinvex on $A$. If $q>1$ and $f^{\prime \prime}$ is integrable on the $\eta$-path $P_{b c}$ for $c=b+\eta(a, b)$, then

$$
\begin{gathered}
\left|\frac{f(b)+f(b+\eta(a, b))}{2}-\frac{1}{\eta(a, b)} \int_{b}^{b+\eta(a, b)} f(x) \mathrm{d} x\right| \\
\leq \frac{[\eta(a, b)]^{2}}{12}\left(\frac{1}{2}\right)^{1 / q}\left[\left|f^{\prime \prime}(a)\right|^{q}+\left|f^{\prime \prime}(b)\right|^{q}\right]^{1 / q} .
\end{gathered}
$$

Recently, some related inequalities for preinvex functions were also obtained in [7, 8]. Some integral inequalities of Hermite-Hadamard type for other kinds of convex functions were also established in [9-16] and references cited therein.

In this paper, by creating an integral identity involving an $n$-times differentiable function, the authors will establish some new Hermite-Hadamard type inequalities for preinvex functions and generalize some of the above mentioned results.

\section{A lemma}

In order to obtain our main results, we need the following lemma.

Lemma 1 For $n \in \mathbb{N}$, let $A \subseteq \mathbb{R}$ be an open invex set with respect to $\eta: A \times A \rightarrow \mathbb{R}$ and let $a, b \in A$ with $\eta(a, b) \neq 0$ for all $a \neq b$. Iff $: A \rightarrow \mathbb{R}$ is an $n$-times differentiable function on $A$ and $f^{(n)}$ is integrable on the $\eta$-path $P_{b c}$ for $c=b+\eta(a, b)$, then

$$
\begin{aligned}
& \frac{f(b)+f(b+\eta(a, b))}{2}-\frac{1}{\eta(a, b)} \int_{b}^{b+\eta(a, b)} f(x) \mathrm{d} x \\
& \quad+\sum_{k=1}^{n-1} \frac{[\eta(a, b)]^{k}(1-k)}{4[(k+1) !]}\left[f^{(k)}(b)+(-1)^{k} f^{(k)}(b+\eta(a, b))\right] \\
& =\frac{[\eta(a, b)]^{n}}{4(n !)} \int_{0}^{1}\left[(1-t)^{n-1}(2 t+n-2)+(-t)^{n-1}(2 t-n)\right] f^{(n)}(b+t \eta(a, b)) \mathrm{d} t,
\end{aligned}
$$

where the above summation is zero for $n=1$.

Proof Since $a, b \in A$ and $A$ is an invex set with respect to $\eta$, for every $t \in[0,1]$, we have $b+t \eta(a, b) \in A$. When $n=1$, integrating by parts in the right-hand side of (1) gives

$$
\frac{f(b)+f(b+\eta(a, b))}{2}-\frac{1}{\eta(a, b)} \int_{b}^{b+\eta(a, b)} f(x) \mathrm{d} x=\frac{\eta(a, b)}{2} \int_{0}^{1}(2 t-1) f^{\prime}(b+t \eta(a, b)) \mathrm{d} t .
$$

Hence, the identity (1) holds for $n=1$.

When $n=m-1$ and $m \geq 2$, suppose that the identity (1) is valid.

When $n=m$, by the hypothesis, we have

$$
\begin{aligned}
& \frac{[\eta(a, b)]^{m}}{4(m !)} \int_{0}^{1}\left[(1-t)^{m-1}(2 t+m-2)+(-t)^{m-1}(2 t-m)\right] f^{(m)}(b+t \eta(a, b)) \mathrm{d} t \\
& =\frac{[\eta(a, b)]^{m-1}}{4(m !)}\left\{(-1)^{m-1}(2-m) f^{(m-1)}(b+\eta(a, b))-(m-2) f^{(m-1)}(b)\right. \\
& \left.\quad-m \int_{0}^{1}\left[(1-t)^{m-2}(3-2 t-m)+(-t)^{m-2}(m-1-2 t)\right] f^{(m-1)}(b+t \eta(a, b)) \mathrm{d} t\right\}
\end{aligned}
$$




$$
\begin{aligned}
= & \frac{[\eta(a, b)]^{m-1}(2-m)}{4(m !)}\left[f^{(m-1)}(b)+(-1)^{m-1} f^{(m-1)}(b+\eta(a, b))\right] \\
& +\frac{[\eta(a, b)]^{m-1}}{4[(m-1) !]} \int_{0}^{1}\left[(1-t)^{m-2}(2 t+m-3)\right. \\
& \left.+(-t)^{m-2}(2 t-m+1)\right] f^{(m-1)}(b+t \eta(a, b)) \mathrm{d} t \\
= & \frac{f(b)+f(b+\eta(a, b))}{2}-\frac{1}{\eta(a, b)} \int_{b}^{b+\eta(a, b)} f(x) \mathrm{d} x \\
& +\sum_{k=1}^{m-1} \frac{[\eta(a, b)]^{k}(1-k)}{4[(k+1)] !}\left[f^{(k)}(b)+(-1)^{k} f^{(k)}(b+\eta(a, b))\right] .
\end{aligned}
$$

Therefore, when $n=m$, the identity (1) holds. By induction, the proof of Lemma 1 is complete.

Remark 1 When $n=1$ and $n=2$ in (1), respectively, we obtain the identities

$$
\begin{gathered}
\frac{f(b)+f(b+\eta(a, b))}{2}-\frac{1}{\eta(a, b)} \int_{b}^{b+\eta(a, b)} f(x) \mathrm{d} x \\
=\frac{\eta(a, b)}{2} \int_{0}^{1}(2 t-1) f^{\prime}(b+t \eta(a, b)) \mathrm{d} t
\end{gathered}
$$

and

$$
\begin{gathered}
\frac{f(b)+f(b+\eta(a, b))}{2}-\frac{1}{\eta(a, b)} \int_{b}^{b+\eta(a, b)} f(x) \mathrm{d} x \\
=\frac{[\eta(a, b)]^{2}}{2} \int_{0}^{1} t(1-t) f^{\prime \prime}(b+t \eta(a, b)) \mathrm{d} t,
\end{gathered}
$$

which may be found in [2].

\section{Hermite-Hadamard type inequalities for preinvex functions}

Now we start out to establish some new Hermite-Hadamard type inequalities for $n$-times differentiable and preinvex functions.

Theorem 7 For $n \in \mathbb{N}$ and $n \geq 2$, let $A \subseteq \mathbb{R}$ be an open invex set with respect to $\eta: A \times$ $A \rightarrow \mathbb{R}$ and $a, b \in A$ with $\eta(a, b) \neq 0$ for all $a \neq b$. Suppose that $f: A \rightarrow \mathbb{R}$ is an n-times differentiable function on $A$ and $f^{(n)}$ is integrable on the $\eta$-path $P_{b c}$ for $c=b+\eta(a, b)$. If $\left|f^{(n)}\right|^{q}$ is preinvex on $A$ for $q \geq 1$, then

$$
\begin{aligned}
& \mid \frac{f(b)+f(b+\eta(a, b))}{2}-\frac{1}{\eta(a, b)} \int_{b}^{b+\eta(a, b)} f(x) \mathrm{d} x \\
& \quad+\sum_{k=1}^{n-1} \frac{[\eta(a, b)]^{k}(1-k)}{4[(k+1) !]}\left[f^{(k)}(b)+(-1)^{k} f^{(k)}(b+\eta(a, b))\right] \mid \\
& \leq \frac{|\eta(a, b)|^{n}(n-1)^{1-1 / q}}{4[(n+1) !](n+2)^{1 / q}}\left\{\left[n\left|f^{(n)}(a)\right|^{q}+\left(n^{2}-2\right)\left|f^{(n)}(b)\right|^{q}\right]^{1 / q}\right. \\
& \left.\quad+\left[\left(n^{2}-2\right)\left|f^{(n)}(a)\right|^{q}+n\left|f^{(n)}(b)\right|^{q}\right]^{1 / q}\right\} .
\end{aligned}
$$


Proof Since $a, b \in A$ and $A$ is an invex set with respect to $\eta$, for every $t \in[0,1]$, we have $b+t \eta(a, b) \in A$. Using Lemma 1 and Hölder's inequality yields

$$
\begin{aligned}
& \frac{f(b)+f(b+\eta(a, b))}{2}-\frac{1}{\eta(a, b)} \int_{b}^{b+\eta(a, b)} f(x) \mathrm{d} x \\
& +\sum_{k=1}^{n-1} \frac{[\eta(a, b)]^{k}(1-k)}{4[(k+1) !]}\left[f^{(k)}(b)+(-1)^{k} f^{(k)}(b+\eta(a, b))\right] \\
& \leq \frac{|\eta(a, b)|^{n}}{4(n !)}\left[\int_{0}^{1}(1-t)^{n-1}(2 t+n-2)\left|f^{(n)}(b+t \eta(a, b))\right| \mathrm{d} t\right. \\
& \left.+\int_{0}^{1} t^{n-1}(n-2 t)\left|f^{(n)}(b+t \eta(a, b))\right| \mathrm{d} t\right] \\
& \leq \frac{|\eta(a, b)|^{n}}{4(n !)}\left\{\left[\int_{0}^{1}(1-t)^{n-1}(2 t+n-2) \mathrm{d} t\right]^{1-1 / q}\right. \\
& \times\left[\int_{0}^{1}(1-t)^{n-1}(2 t+n-2)\left(t\left|f^{(n)}(a)\right|^{q}+(1-t)\left|f^{(n)}(b)\right|^{q}\right) \mathrm{d} t\right]^{1 / q} \\
& +\left[\int_{0}^{1} t^{n-1}(n-2 t) \mathrm{d} t\right]^{1-1 / q}\left[\int _ { 0 } ^ { 1 } t ^ { n - 1 } ( n - 2 t ) \left(t\left|f^{(n)}(a)\right|^{q}\right.\right. \\
& \left.\left.\left.+(1-t)\left|f^{(n)}(b)\right|^{q}\right) \mathrm{~d} t\right]^{1 / q}\right\} \\
& =\frac{|\eta(a, b)|^{n}(n-1)^{1-1 / q}}{4[(n+1) !](n+2)^{1 / q}}\left\{\left[n\left|f^{(n)}(a)\right|^{q}+\left(n^{2}-2\right)\left|f^{(n)}(b)\right|^{q}\right]^{1 / q}\right. \\
& \left.+\left[\left(n^{2}-2\right)\left|f^{(n)}(a)\right|^{q}+n\left|f^{(n)}(b)\right|^{q}\right]^{1 / q}\right\} .
\end{aligned}
$$

Theorem 7 is thus proved.

\section{Corollary 1 Under the assumptions of Theorem 7,}

1. if $q=1$, then

$$
\begin{aligned}
& \mid \frac{f(b)+f(b+\eta(a, b))}{2}-\frac{1}{\eta(a, b)} \int_{b}^{b+\eta(a, b)} f(x) \mathrm{d} x \\
& \quad+\sum_{k=1}^{n-1} \frac{[\eta(a, b)]^{k}(1-k)}{4[(k+1) !]}\left[f^{(k)}(b)+(-1)^{k} f^{(k)}(b+\eta(a, b))\right] \mid \\
& \quad \leq \frac{(n-1)|\eta(a, b)|^{n}}{4[(n+1) !]}\left[\left|f^{(n)}(a)\right|+\left|f^{(n)}(b)\right|\right] ;
\end{aligned}
$$

2. if $q=1$ and $n=2$, then the inequality (8) is valid.

Theorem 8 For $n \in \mathbb{N}$ and $n \geq 2$, let $A \subseteq \mathbb{R}$ be an open invex set with respect to $\eta: A \times$ $A \rightarrow \mathbb{R}$ and $a, b \in A$ with $\eta(a, b) \neq 0$ for all $a \neq b$. Suppose that $f: A \rightarrow \mathbb{R}$ is an n-times differentiable function on $A$ and $f^{(n)}$ is integrable on the $\eta$-path $P_{b c}$ for $c=b+\eta(a, b)$. If 
$\left|f^{(n)}\right|^{q}$ is preinvex on $A$ for $q>1$, then

$$
\begin{aligned}
& \mid \frac{f(b)+f(b+\eta(a, b))}{2}-\frac{1}{\eta(a, b)} \int_{b}^{b+\eta(a, b)} f(x) \mathrm{d} x \\
& +\frac{1}{4} \sum_{k=1}^{n-1} \frac{[\eta(a, b)]^{k}(1-k)}{(k+1) !}\left[f^{(k)}(b)+(-1)^{k} f^{(k)}(b+\eta(a, b))\right] \\
& \leq \frac{|\eta(a, b)|^{n}}{16(n !)[(q+1)(q+2)]^{1 / q}}\left[\frac{4(q-1)}{n q-1}\right]^{1-1 / q} \\
& \times\left\{\left[\left((n-2)^{q+2}-(n-2 q-4) n^{q+1}\right)\left|f^{(n)}(a)\right|^{q}\right.\right. \\
& \left.+\left(n^{q+2}-(n+2 q+2)(n-2)^{q+1}\right)\left|f^{(n)}(b)\right|^{q}\right]^{1 / q} \\
& +\left[\left(n^{q+2}-(n+2 q+2)(n-2)^{q+1}\right)\left|f^{(n)}(a)\right|^{q}\right. \\
& \left.\left.+\left((n-2)^{q+2}-(n-2 q-4) n^{q+1}\right)\left|f^{(n)}(b)\right|^{q}\right]^{1 / q}\right\} .
\end{aligned}
$$

Proof For every $t \in[0,1]$, we have $b+t \eta(a, b) \in A$. By Lemma 1 and Hölder's inequality, it follows that

$$
\begin{aligned}
& \frac{f(b)+f(b+\eta(a, b))}{2}-\frac{1}{\eta(a, b)} \int_{b}^{b+\eta(a, b)} f(x) \mathrm{d} x \\
& +\sum_{k=1}^{n-1} \frac{[\eta(a, b)]^{k}(1-k)}{4[(k+1) !]}\left[f^{(k)}(b)+(-1)^{k} f^{(k)}(b+\eta(a, b))\right] \\
& \leq \frac{|\eta(a, b)|^{n}}{4(n !)}\left[\int_{0}^{1}(1-t)^{n-1}(2 t+n-2)\left|f^{(n)}(b+t \eta(a, b))\right| \mathrm{d} t\right. \\
& \left.+\int_{0}^{1} t^{n-1}(n-2 t)\left|f^{(n)}(b+t \eta(a, b))\right| \mathrm{d} t\right] \\
& \leq \frac{|\eta(a, b)|^{n}}{4(n !)}\left\{\left[\int_{0}^{1}(1-t)^{q(n-1) /(q-1)} \mathrm{d} t\right]^{1-1 / q}\right. \\
& \times\left[\int_{0}^{1}(2 t+n-2)^{q}\left(t\left|f^{(n)}(a)\right|^{q}+(1-t)\left|f^{(n)}(b)\right|^{q}\right) \mathrm{d} t\right]^{1 / q} \\
& \left.+\left[\int_{0}^{1} t^{q(n-1) /(q-1)} \mathrm{d} t\right]^{1-1 / q}\left[\int_{0}^{1}(n-2 t)^{q}\left(t\left|f^{(n)}(a)\right|^{q}+(1-t)\left|f^{(n)}(b)\right|^{q}\right) \mathrm{d} t\right]^{1 / q}\right\} \\
& =\frac{|\eta(a, b)|^{n}}{16(n !)[(q+1)(q+2)]^{1 / q}}\left[\frac{4(q-1)}{n q-1}\right]^{1-1 / q} \\
& \times\left\{\left[\left((n-2)^{q+2}-(n-2 q-4) n^{q+1}\right)\left|f^{(n)}(a)\right|^{q}\right.\right. \\
& \left.+\left(n^{q+2}-(n+2 q+2)(n-2)^{q+1}\right)\left|f^{(n)}(b)\right|^{q}\right]^{1 / q} \\
& +\left[\left(n^{q+2}-(n+2 q+2)(n-2)^{q+1}\right)\left|f^{(n)}(a)\right|^{q}\right. \\
& \left.\left.+\left((n-2)^{q+2}-(n-2 q-4) n^{q+1}\right)\left|f^{(n)}(b)\right|^{q}\right]^{1 / q}\right\} .
\end{aligned}
$$

Theorem 8 is thus proved. 
Theorem 9 For $n \in \mathbb{N}$ and $n \geq 2$, let $A \subseteq \mathbb{R}$ be an open invex set with respect to $\eta: A \times$ $A \rightarrow \mathbb{R}$ and $a, b \in A$ with $\eta(a, b) \neq 0$ for all $a \neq b$. Suppose that $f: A \rightarrow \mathbb{R}$ is an n-times differentiable function on $A$ and $f^{(n)}$ is integrable on the $\eta$-path $P_{b c}$ for $c=b+\eta(a, b)$. If $\left|f^{(n)}\right|^{q}$ is preinvex on $A$ for $q>1$, then

$$
\begin{aligned}
& \frac{f(b)+f(b+\eta(a, b))}{2}-\frac{1}{\eta(a, b)} \int_{b}^{b+\eta(a, b)} f(x) \mathrm{d} x \\
& +\sum_{k=1}^{n-1} \frac{[\eta(a, b)]^{k}(1-k)}{4[(k+1) !]}\left[f^{(k)}(b)+(-1)^{k} f^{(k)}(b+\eta(a, b))\right] \\
& \leq \frac{|\eta(a, b)|^{n}}{4(n !)[(n q-q+1)(n q-q+2)]^{1 / q}} \\
& \times\left\{\frac{(q-1)\left[n^{(2 q-1) /(q-1)}-(n-2)^{(2 q-1) /(q-1)}\right]}{2(2 q-1)}\right\}^{1-1 / q} \\
& \times\left\{\left[\left|f^{(n)}(a)\right|^{q}+(n q-q+1)\left|f^{(n)}(b)\right|^{q}\right]^{1 / q}\right. \\
& \left.+\left[(n q-q+1)\left|f^{(n)}(a)\right|^{q}+\left|f^{(n)}(b)\right|^{q}\right]^{1 / q}\right\} .
\end{aligned}
$$

Proof Since $a, b \in A$ and $A$ is an invex set with respect to $\eta$, for every $t \in[0,1]$, we have $b+t \eta(a, b) \in A$. Utilizing Lemma 1 and Hölder's inequality results in

$$
\begin{aligned}
& \frac{f(b)+f(b+\eta(a, b))}{2}-\frac{1}{\eta(a, b)} \int_{b}^{b+\eta(a, b)} f(x) \mathrm{d} x \\
& +\sum_{k=1}^{n-1} \frac{[\eta(a, b)]^{k}(1-k)}{4[(k+1) !]}\left[f^{(k)}(b)+(-1)^{k} f^{(k)}(b+\eta(a, b))\right] \\
& \leq \frac{|\eta(a, b)|^{n}}{4(n !)}\left[\int_{0}^{1}(1-t)^{n-1}(2 t+n-2)\left|f^{(n)}(b+t \eta(a, b))\right| \mathrm{d} t\right. \\
& \left.+\int_{0}^{1} t^{n-1}(n-2 t)\left|f^{(n)}(b+t \eta(a, b))\right| \mathrm{d} t\right] \\
& \leq \frac{|\eta(a, b)|^{n}}{4(n !)}\left\{\left[\int_{0}^{1}(2 t+n-2)^{q /(q-1)} \mathrm{d} t\right]^{1-1 / q}\right. \\
& \times\left[\int_{0}^{1}(1-t)^{q(n-1)}\left(t\left|f^{(n)}(a)\right|^{q}+(1-t)\left|f^{(n)}(b)\right|^{q}\right) \mathrm{d} t\right]^{1 / q} \\
& \left.+\left[\int_{0}^{1}(n-2 t)^{q /(q-1)} \mathrm{d} t\right]^{1-1 / q}\left[\int_{0}^{1} t^{q(n-1)}\left(t\left|f^{(n)}(a)\right|^{q}+(1-t)\left|f^{(n)}(b)\right|^{q}\right) \mathrm{d} t\right]^{1 / q}\right\} \\
& =\frac{|\eta(a, b)|^{n}}{4(n !)[(n q-q+1)(n q-q+2)]^{1 / q}} \\
& \times\left\{\frac{(q-1)\left[n^{(2 q-1) /(q-1)}-(n-2)^{(2 q-1) /(q-1)}\right]}{2(2 q-1)}\right\}^{1-1 / q} \\
& \times\left\{\left[\left|f^{(n)}(a)\right|^{q}+(n q-q+1)\left|f^{(n)}(b)\right|^{q}\right]^{1 / q}\right. \\
& \left.+\left[(n q-q+1)\left|f^{(n)}(a)\right|^{q}+\left|f^{(n)}(b)\right|^{q}\right]^{1 / q}\right\} .
\end{aligned}
$$

The proof of Theorem 9 is complete. 
Theorem 10 For $n \in \mathbb{N}$ and $n \geq 2$, let $A \subseteq \mathbb{R}$ be an open invex set with respect to $\eta: A \times$ $A \rightarrow \mathbb{R}$ and $a, b \in A$ with $\eta(a, b) \neq 0$ for all $a \neq b$. Suppose that $f: A \rightarrow \mathbb{R}$ is an n-times differentiable function on $A$ and $f^{(n)}$ is integrable on the $\eta$-path $P_{b c}$ for $c=b+\eta(a, b)$. If $\left|f^{(n)}\right|^{q}$ is preinvex on $A$ for $q>1$, then

$$
\begin{aligned}
& \mid \frac{f(b)+f(b+\eta(a, b))}{2}-\frac{1}{\eta(a, b)} \int_{b}^{b+\eta(a, b)} f(x) \mathrm{d} x \\
& +\frac{1}{4} \sum_{k=1}^{n-1} \frac{[\eta(a, b)]^{k}(1-k)}{(k+1) !}\left[f^{(k)}(b)+(-1)^{k} f^{(k)}(b+\eta(a, b))\right] \\
& \leq \frac{|\eta(a, b)|^{n}}{24(n !)}\left[\frac{6(q-1)(n q-2)(n-1)}{(n q-1)(n q+q-2)}\right]^{1-1 / q} \\
& \times\left\{\left[(3 n-2)\left|f^{(n)}(a)\right|^{q}+(3 n-4)\left|f^{(n)}(b)\right|^{q}\right]^{1 / q}\right. \\
& \left.+\left[(3 n-4)\left|f^{(n)}(a)\right|^{q}+(3 n-2)\left|f^{(n)}(b)\right|^{q}\right]^{1 / q}\right\} .
\end{aligned}
$$

Proof Since $a, b \in A$ and $A$ is an invex set with respect to $\eta$, for every $t \in[0,1]$, we have $b+t \eta(a, b) \in A$. Employing Lemma 1 and Hölder's inequality leads to

$$
\begin{aligned}
\mid \frac{f(b)}{}+ & f(b+\eta(a, b)) \\
2 & -\frac{1}{\eta(a, b)} \int_{b}^{b+\eta(a, b)} f(x) \mathrm{d} x \\
& +\sum_{k=1}^{n-1} \frac{[\eta(a, b)]^{k}(1-k)}{4[(k+1) !]}\left[f^{(k)}(b)+(-1)^{k} f^{(k)}(b+\eta(a, b))\right] \mid \\
\leq & \frac{|\eta(a, b)|^{n}}{4(n !)}\left[\int_{0}^{1}(1-t)^{n-1}(2 t+n-2)\left|f^{(n)}(b+t \eta(a, b))\right| \mathrm{d} t\right. \\
& \left.+\int_{0}^{1} t^{n-1}(n-2 t)\left|f^{(n)}(b+t \eta(a, b))\right| \mathrm{d} t\right] \\
\leq & \frac{|\eta(a, b)|^{n}}{4(n !)}\left\{\left[\int_{0}^{1}(1-t)^{q(n-1) /(q-1)}(2 t+n-2) \mathrm{d} t\right]^{1-1 / q}\right. \\
& \times\left[\int_{0}^{1}(2 t+n-2)\left(t\left|f^{(n)}(a)\right|^{q}+(1-t)\left|f^{(n)}(b)\right|^{q}\right) \mathrm{d} t\right]^{1 / q} \\
& +\left[\int_{0}^{1} t^{q(n-1) /(q-1)}(n-2 t) \mathrm{d} t\right]^{1-1 / q}\left[\int _ { 0 } ^ { 1 } ( n - 2 t ) \left(t\left|f^{(n)}(a)\right|^{q}\right.\right. \\
& \left.\left.\left.+(1-t)\left|f^{(n)}(b)\right|^{q}\right) \mathrm{~d} t\right]^{1 / q}\right\} \\
= & \frac{|\eta(a, b)|^{n}}{24(n !)}\left[\frac{6(q-1)(n q-2)(n-1)}{(n q-1)(n q+q-2)}\right]^{1-1 / q}\left\{\left[(3 n-2)\left|f^{(n)}(a)\right|^{q}\right.\right. \\
& \left.\left.+(3 n-4)\left|f^{(n)}(b)\right|^{q}\right]^{1 / q}+\left[(3 n-4)\left|f^{(n)}(a)\right|^{q}+(3 n-2)\left|f^{(n)}(b)\right|^{q}\right]^{1 / q}\right\} .
\end{aligned}
$$

The proof of Theorem 10 is complete. 
Authors' contributions

All authors contributed equally to the manuscript and read and approved the final manuscript.

\section{Author details}

'College of Mathematics, Inner Mongolia University for Nationalities, Tongliao City, Inner Mongolia Autonomous Region 028043, China. ${ }^{2}$ Department of Mathematics, College of Science, Tianjin Polytechnic University, Tianjin City, 300387, China. ${ }^{3}$ Institute of Mathematics, Henan Polytechnic University, Jiaozuo City, 454010, China.

\section{Acknowledgements}

The authors appreciate anonymous referees for their valuable comments on and careful corrections to the original version of this paper. This work was partially supported by the NNSF under Grant No. 11361038 of China and by the Foundation of the Research Program of Science and Technology at Universities of Inner Mongolia Autonomous Region under Grant No. NJZY13159, China.

Received: 29 August 2013 Accepted: 8 January 2014 Published: 30 Jan 2014

\section{References}

1. Antczak, T: Mean value in invexity analysis. Nonlinear Anal. 60(8), 1471-1484 (2005). doi:10.1016/j.na.2004.11.005

2. Barani, A, Ghazanfariand, AG, Dragomir, SS: Hermite-Hadamard inequality for functions whose derivatives absolute values are preinvex. J. Inequal. Appl. 2012, 247 (2012). doi:10.1186/1029-242X-2012-247

3. Mohan, SR, Neogy, SK: On invex sets and preinvex function. J. Math. Anal. Appl. 189(3), 901-908 (1995). doi:10.1006/jmaa.1995.1057

4. Dragomir, SS, Agarwal, RP: Two inequalities for differentiable mappings and applications to special means of real numbers and to trapezoidal formula. Appl. Math. Lett. 11(5), 91-95 (1998). doi:10.1016/S0893-9659(98)00086-X

5. Pearce, CEM, Pečarič, J: Inequalities for differentiable mappings with application to special means and quadrature formulae. Appl. Math. Lett. 13(2), 51-55 (2000). doi:10.1016/S0893-9659(99)00164-0

6. Kirmaci, US: Inequalities for differentiable mappings and applications to special means of real numbers to midpoint formula. Appl. Math. Comput. 147(1), 137-146 (2004). doi:10.1016/S0096-3003(02)00657-4

7. Wang, Y, Wang, S-H, Qi, F: Simpson type integral inequalities in which the power of the absolute value of the first derivative of the integrand is s-preinvex. Facta Univ., Ser. Math. Inform. 28(2), 151-159 (2013)

8. Wang, Y, Xi, B-Y, Qi, F: Hermite-Hadamard type integral inequalities when the power of the absolute value of the first derivative of the integrand is preinvex. Matematiche 69(1) (2014, in press)

9. Chun, L, Qi, F: Integral inequalities of Hermite-Hadamard type for functions whose third derivatives are convex. J. Inequal. Appl. 2013, 451 (2013). doi:10.1186/1029-242X-2013-451

10. Li, W-H, Qi, F: Some Hermite-Hadamard type inequalities for functions whose $n$-th derivatives are $(\alpha, m)$-convex. Filomat 27(8), 1575-1582 (2013). doi:10.2298/FIL1308575L

11. Qi, F, Xi, B-Y: Some integral inequalities of Simpson type for GA- $\varepsilon$-convex functions. Georgian Math. J. 20(4), 775-788 (2013). doi:10.1515/gmj-2013-0043

12. Shuang, Y, Wang, Y, Qi, F: Some inequalities of Hermite-Hadamard type for functions whose third derivatives are $(\alpha, m)$-convex. J. Comput. Anal. Appl. 17(2), 272-279 (2014)

13. Xi, B-Y, Bai, R-F, Qi, F: Hermite-Hadamard type inequalities for the $m$ - and $(\alpha, m)$-geometrically convex functions. Aequ. Math. 84(3), 261-269 (2012). doi:10.1007/s00010-011-0114-X

14. Xi, B-Y, Qi, F: Some Hermite-Hadamard type inequalities for differentiable convex functions and applications. Hacet. J. Math. Stat. 42(3), 243-257 (2013)

15. Xi, B-Y, Qi, F: Some integral inequalities of Hermite-Hadamard type for convex functions with applications to means. J. Funct. Spaces Appl. 2012, Article ID 980438 (2012). doi:10.1155/2012/980438

16. Zhang, B, Xi, B-Y, Qi, F: Some properties and inequalities for $h$-geometrically convex functions. J. Classical Anal. 3(2), 101-108 (2014). doi:10.7153/jca-03-09

10.1186/1029-242X-2014-49

Cite this article as: Wang and Qi: Hermite-Hadamard type inequalities for $n$-times differentiable and preinvex functions. Journal of Inequalities and Applications 2014, 2014:49

\section{Submit your manuscript to a SpringerOpen ${ }^{\odot}$ journal and benefit from:}

- Convenient online submission

- Rigorous peer review

- Immediate publication on acceptance

Open access: articles freely available online

- High visibility within the field

- Retaining the copyright to your article 